\title{
ANDES

www.scielo.cl

\section{Características del tratamiento recibido por los niños con fisura labio alvéolo palatina en Argentina}

\author{
Characteristics of the treatment received by children with cleft lip and palate \\ in Argentina
}

\author{
María C. Cipolla ${ }^{\mathrm{a}}$, Agustina Piola ${ }^{\mathrm{b}}$, Pablo Barbero ${ }^{\mathrm{b}}$, Boris Groisman ${ }^{\mathrm{b}}$, \\ María P. Bidondo ${ }^{\mathrm{b}}$, Roberto Chuit ${ }^{\mathrm{c}}$, Rosa Liascovich ${ }^{\mathrm{b}}$
}

aDirección de Salud Perinatal y Niñez, Ministerio de Salud. Buenos Aires, Argentina

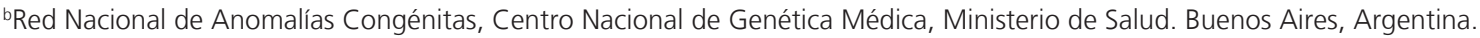

Instituto de Investigaciones Epidemiológicas, Academia Nacional de Medicina. Buenos Aires, Argentina.

Recibido: 10 de julio de 2020; Aceptado: 02 de octubre de 2020

\section{¿Qué se sabe del tema que trata este estudio?}

En Argentina, existen publicaciones sobre los resultados quirúrgicos a corto plazo del tratamiento de los niños fisurados, pero poco se sabe sobre la evolución, en el mediano plazo, en términos del seguimiento interdisciplinario e integral de la enfermedad.

\begin{abstract}
¿Qué aporta este estudio a lo ya conocido?
Esta investigación es la primera en Argentina que aporta información sobre la evolución en los primeros años de la niñez, de los niños nacidos con fisuras orales aisladas, asistidos en los servicios de la Red Flap, de los efectores públicos. Propone además un modelo de indicadores para la evaluación del tratamiento, en términos de oportunidad, interdisciplina e integralidad.
\end{abstract}

\section{Resumen}

Las fisuras labio palatinas (FLAP) son anomalías congénitas que afectan anatómica y funcionalmente la cara y boca, comprometiendo labio (FL), paladar (FP), o ambos (FL/FP). Objetivo: caracterizar el tratamiento de niños con FLAP en instituciones públicas en Argentina. Pacientes y Método: Estudio transversal en muestra aleatoria de 100 niños de la Red Flap. Se incluyeron niños con FL, FP y FL/FP aisladas, de ambos sexos, con peso nacimiento de 2500 gramos o más y edad gestacional mayor a 36 semanas. Se reportó fecha de nacimiento, hospital de nacimiento, peso nacimiento, edad gestacional, sexo, diagnóstico específico de la fisura, y datos de la cirugía inicial. Se realizó una encuesta telefónica a los progenitores de los niños. Para caracterizar el tratamiento se construyeron tres indicadores: interdisciplina, oportunidad e integralidad, que se compusieron de diferentes variables y según la suma del puntaje atribuido a cada una, se categorizó en: alto, medio o bajo según las pautas de tratamiento que usa el Programa Sumar. Resultados: Iniciaron tratamiento precoz el 30\%. El 58\% se operó opor-
Palabras clave: Fisura de Paladar; Fisura de Labio; Fisura palatina; Tratamiento; Argentina 
tunamente e hicieron seguimiento con las especialidades básicas el 29\% de los niños. El alto nivel educativo materno se asoció con mayor probabilidad de tener tratamiento interdisciplinario (OR2,9; IC95\% 1,3-6,8), integral (OR3,7; IC95\% 1,6-8,7) y oportuno (OR2,9; IC95\% 1,3-6,7). Conclusiones: Existen barreras para acceder a la atención, como las grandes distancias o la gestión de los turnos. La menor vulnerabilidad social de las familias se asoció con mayor probabilidad de recibir un tratamiento cercano a los estándares.

\begin{abstract}
Cleft lip and palate (CLP) are congenital abnormalities that affect anatomically and functionally the face and mouth, involving lip (CL), palate (CP), or both (CL/CP). Objective: to characterize the treatment of children with CLP in public institutions in Argentina. Patients and Method: Crosssectional study in a random sample of 100 children from the Flap Network. We included children with isolated CL, CP, and CL/CP, of both sexes, with birth weight of 2500 grams or more and gestational age over 36 weeks. The following data were recorded date of birth, hospital of birth, birth weight, gestational age, sex, specific diagnosis of the cleft, and initial surgery data. A telephone survey was conducted with the children's parents. To characterize the treatment, three indicators were constructed: interdisciplinary, opportunity, and integrality. These indicators were composed of different variables, and according to the sum of the score attributed to each one, the treatment was categorized as high, medium or low based on the treatment guidelines used by the Sumar Program. Results: $30 \%$ of the patients started early treatment, $58 \%$ underwent timely surgery, and $29 \%$ of the children were in follow-up with basic specialties. The mother's high educational level was associated with higher probability of having interdisciplinary (OR2.9; 95\%CI 1.3-6.8), comprehensive (OR3.7; 95\%CI 1.68.7), and timely treatment (OR2.9; 95\%CI 1.3-6.7). Conclusions: There are barriers to accessing care, such as long distances or shift management. Less social vulnerability of families was associated with greater likelihood of receiving treatment close to standards.
\end{abstract}

\section{Keywords:}

Cleft Palate;

Cleft Lip;

Treatment;

Argentina

\section{Introducción}

Las fisuras labio alvéolo palatinas (FLAP) son anomalías congénitas cráneo faciales que afectan la anatomía y funcionalidad de la cara y la boca y pueden comprometer el labio (FL), el paladar (FP) o a ambos (FL/FP). La etiología de las FLAP es multifactorial, con factores genéticos predisponentes y factores ambientales desencadenantes. El factor de riesgo más conocido es el tabaquismo materno durante la gestación ${ }^{1,2}$. También se ha estudiado el consumo materno de alcohol durante el embarazo ${ }^{3}$, la edad avanzada de los padres ${ }^{4,5}$, la consanguinidad ${ }^{6}$ y el nivel socioeconómico bajo, entre otros factores ${ }^{7,8}$. Algunos trabajos encontraron que el consumo prenatal de ácido fólico es un factor protector?.

La prevalencia de las FLAP al nacimiento es heterogénea: alta entre asiáticos y amerindios ( 8 a 37 cada 10.000 nacidos vivos), intermedia en caucási$\cos (9$ a 27 cada 10.000) y baja en africanos (2 a 17 cada 10.000$)^{10}$. La prevalencia de niños con FLAP en Argentina en el año 2018 fue de 16,85 (15,32-18,49) cada 10.000 nacimientos, por lo que se estiman unos 1.100 a 1.200 nuevos afectados cada año ${ }^{11}$. Las fisuras orales pueden presentarse en forma aislada, asociadas con otras anomalías congénitas o formando parte de síndromes. Algunas fisuras orales pueden detectarse por ultrasonografía prenatal, especialmente cuando se presentan acompañadas de otras anomalías. En general la malformación no conlleva riesgo de muerte, pero la morbilidad es mayor que en los niños no afec$\operatorname{tados}^{12}$. Las principales comorbilidades en los primeros años de vida son las patologías fonoaudiológicas y odontológicas, y luego, sus posibles consecuencias: trastornos del lenguaje, de aprendizaje y bajo rendimiento escolar ${ }^{13,14}$. Por eso el tratamiento de un niño con FLAP es complejo, requiere varios años y múltiples intervenciones quirúrgicas y no quirúrgicas, desde el nacimiento hasta la adolescencia. Como en la mayoría de las enfermedades crónicas de los niños, existen barreras organizacionales y geográficas que dificultan el acceso al tratamiento. Todas las recomendaciones locales e internacionales coinciden en que el tratamiento debe ser interdisciplinario, oportuno e integral ${ }^{15-18}$.

Como antecedente, en el año 2006 con el objeto de obtener información local sobre los resultados del tratamiento de los niños fisurados, se organizó un trabajo en red de diferentes servicios de rehabilitación de pacientes con FLAP (públicos y privados) en el ámbito nacional ${ }^{19}$.

En 2009 a fin de establecer un sistema nacional de 
vigilancia de defectos congénitos, se creó en Argentina el Registro (luego Red) Nacional de Anomalías Congénitas (RENAC), cuya coordinación depende del Centro Nacional de Genética Médica (Administración Nacional de Laboratorios e Institutos de Salud), del Ministerio de Salud. La cobertura de la RENAC es del $62 \%$ de los nacimientos del subsector público y el $43 \%$ del total de nacimientos de Argentina. A partir de 2015 la RENAC fue centro coordinador de la "Red Federal para la Atención de niños/as con FLAP" (en adelante "Red Flap"), creada en alianza con el Programa Sumar ${ }^{\star}$ para fortalecer las prácticas de cuidado de los niños que poseen exclusivamente cobertura pública de salud. Al momento del presente trabajo las instituciones acreditadas son 64 efectores públicos distribuidos en todas las provincias del país, excepto Tierra del Fuego. La mayoría tiene equipo profesional completo, es decir, cuenta con las tres especialidades consideradas básicas: fonoaudiología, odontología y cirugía (FOC); del resto, gran parte lo completa en otra institución de su localidad o de otra localidad, y una pequeña parte no lo completa.

Al momento, sólo se ha publicado un trabajo que muestra la organización preliminar y funcionamiento de la Red Flap ${ }^{20}$, pero no hay datos a nivel país sobre el tratamiento de los niños con fisuras orales del subsector público. El objetivo del presente estudio fue describir las características del tratamiento de los niños con FL, FP o FL/FP aisladas, asistidos en las instituciones de la Red Flap en Argentina nacidos durante 2015 y 2016.

\section{Pacientes y Método}

La RENAC incluye unas 150 maternidades de todas las provincias argentinas. En cada maternidad dos referentes pediatras o neonatólogos reportan mensualmente los recién nacidos (RN) con anomalías congénitas mayores identificadas desde el nacimiento hasta el alta hospitalaria. En el caso de los recién nacidos con FLAP, además se envía una notificación dentro de las primeras $48 \mathrm{~h}$ de vida, se gestiona la derivación y se planifica el seguimiento y tratamiento con las instituciones tratantes acreditadas por la Red Flap según el lugar de residencia de las familias. Además de las especialidades básicas (FOC), se incluye un plan de interconsultas con otorrinolaringología, servicio social,

${ }^{*}$ El Sumar es un programa del Ministerio de Salud de la Argentina, que nominaliza a la población sin cobertura de obra social y paga por el desempeño de ciertas acciones priorizadas a las provincias. A través del Sumar, la Nación transfiere recursos a las provincias en función del cumplimiento de metas sanitarias. Con los recursos obtenidos, las provincias financian prestaciones de salud brindadas a la población en los establecimientos públicos. psicología, genética, y cardiología, según el caso, coordinado por un pediatra.

El presente estudio tiene un enfoque cuantitativo, es observacional-analítico, de corte transversal. La unidad de análisis es el niño y su familia.

Criterio de inclusión: niños con FL, FP y FL/FP aisladas, de ambos sexos, con peso de nacimiento de 2500 gramos o más y edad gestacional mayor a 36 semanas.

Criterio de exclusión: niños con FLAP asociadas a otras anomalías congénitas o con cobertura de obra social o privada al nacer.

Se seleccionó una muestra aleatoria, representativa de la población siguiendo los criterios de inclusión. La muestra resultó en una prevalencia estimada del 50\%, precisión del $5 \%$ y un nivel de confianza del $95 \%$, por lo cual fue necesario incluir 100 niños. Los casos se seleccionaron del listado de números aleatorios de la población de referencia de $623 \mathrm{RN}$ con FLAP, reportados entre el $1^{\circ}$ de enero de 2015 y el 31 de diciembre de 2016 en maternidades de la RENAC. En caso de no aceptar o no cumplir con los criterios de inclusión, el niño seleccionado fue reemplazado por el registro anterior y/o posterior hasta encontrar uno válido. Los reportes existentes en la Red Flap (fuente secundaria) aportaron: fecha de nacimiento, hospital de nacimiento, peso al nacer, edad gestacional, sexo, datos filiatorios del niño y su madre, diagnóstico específico de la fisura, nombre de la institución de derivación y realización de la primera cirugía. Se realizó una encuesta telefónica (fuente primaria) a los progenitores de los niños, que incluyó un cuestionario estructurado de 30 preguntas cerradas confeccionado ad hoc, e insumió entre 13 y 22 minutos. Para las variables sociales se utilizaron preguntas validadas de los cuestionarios que utiliza el Instituto Nacional de Estadísticas y Censo ${ }^{21}$; para las variables del control prenatal se utilizaron las del Sistema Informático Perinatal ${ }^{22}$ y para las variables de barreras geográficas se utilizaron las preguntas validadas de un estudio publicado de niños con FLAP ${ }^{23}$.

Todas las encuestas fueron realizadas entre agosto 2018 y mayo de 2019, por la misma persona; sólo hubo dos rechazos a participar. La encuesta fue respondida por uno de los progenitores del niño (90\% madres, $10 \%$ padres).

Para caracterizar el tratamiento se construyeron tres indicadores: interdisciplina, oportunidad e integralidad (Ver Anexo). Cada indicador se compuso de diferentes variables y según la suma del puntaje atribuido a cada una, se categorizó en: alto, medio o bajo según las pautas de tratamiento que usa el Programa Sumar ${ }^{15}$.

Para evaluar las barreras de acceso al tratamiento se conformaron dos indicadores, barreras organizacionales y barreras geográficas. El primero consideró estas variables: gestión del turno de la primera consulta 
y gestión de los turnos de consultas ulteriores. Para el indicador de barreras geográficas se consideró el tiempo que la familia habitualmente tarda en llegar desde su casa hasta la institución tratante, cómo se traslada habitualmente y el número de medios de transporte utilizados.

Para analizar qué factores del niño, la familia, o las barreras de acceso, se asociaron con los indicadores del tratamiento, se usó el OR con intervalo de confianza del $95 \%$ y el estadístico de $\chi^{2}$ con un nivel alfa de 0.05 . Para todo el análisis se utilizó el Programa Epi Info 7.2.

El protocolo del estudio fue aprobado por el Comité de Ética del Centro Nacional de Genética Médica. Todos los progenitores entrevistados aceptaron un consentimiento informado que les fue leído previamente, las entrevistas no se grabaron. Se respetó en todo momento el secreto estadístico, velando por el anonimato de la información, según establecen las leyes nacionales de secreto estadístico y de protección datos personales.

\section{Resultados}

La media de edad de los 100 niños al momento de la entrevista fue de 36,2 meses, la mediana de 36,1 y el rango de 21,8 a 49,4 meses. Como diagnóstico de FLAP, 17 niños presentaban FL, 62 FL/FP y 21 FP. Los niños fueron asistidos en 31 instituciones, 20 de las cuales tenían equipo de FLAP completo, dos lo completaban en la misma localidad, uno en otra localidad y dos no completaban equipo. En la tabla 1 se presentan las características sociosanitarias de los niños y sus familias.

Tabla 1. Características sociosanitarias de los niños (y sus familias) asistidos en la Red Flap, Argentina, 2015-2016

\begin{tabular}{|c|c|c|}
\hline Características & Categorías & $n$ \\
\hline Sexo & $\begin{array}{l}\text { Masculino } \\
\text { Femenino }\end{array}$ & $\begin{array}{l}58 \\
42\end{array}$ \\
\hline Año de nacimiento & $\begin{array}{l}2015 \\
2016\end{array}$ & $\begin{array}{l}46 \\
54\end{array}$ \\
\hline Región del país & $\begin{array}{l}\text { Centro } \\
\text { Noroeste } \\
\text { Noreste } \\
\text { Cuyo } \\
\text { Patagonia }\end{array}$ & $\begin{array}{r}49 \\
18 \\
14 \\
13 \\
6\end{array}$ \\
\hline Edad & $\begin{array}{l}\text { Menor de } 24 \text { meses } \\
\text { De } 24 \text { a } 35 \text { meses } \\
\text { De } 36 \text { a } 47 \text { meses } \\
\text { De } 48 \text { meses o más }\end{array}$ & $\begin{array}{r}2 \\
48 \\
47 \\
3\end{array}$ \\
\hline Tipo de fisura & $\begin{array}{l}\text { Labio y Paladar } \\
\text { Paladar } \\
\text { Labio }\end{array}$ & $\begin{array}{l}62 \\
21 \\
17\end{array}$ \\
\hline Control prenatal & $\begin{array}{l}\text { Suficiente (al menos } 5 \text { controles) } \\
\text { Precoz (antes de las } 13 \text { semanas) } \\
\text { Adecuado (suficiente y precoz) }\end{array}$ & $\begin{array}{l}72 \\
72 \\
72\end{array}$ \\
\hline Diagnóstico prenatal de FLAP & $\begin{array}{l}\text { No } \\
\text { Si (FL/FP =24; FL =9; F P=2) }\end{array}$ & $\begin{array}{l}65 \\
35\end{array}$ \\
\hline Conviven madre y padre & $\begin{array}{l}\mathrm{Si} \\
\text { No }\end{array}$ & $\begin{array}{l}74 \\
26\end{array}$ \\
\hline Edad de la madre & $\begin{array}{l}\text { Hasta } 20 \text { años } \\
\text { Entre } 21 \text { y } 39 \text { años } \\
40 \text { años o más }\end{array}$ & $\begin{array}{r}5 \\
84 \\
11\end{array}$ \\
\hline Nivel educativo de la madre y el padre & $\begin{array}{l}\text { Madre con secundario completo o más } \\
\text { Padre con secundario completo o más } \\
\text { Madre y padre con secundario completo o más }\end{array}$ & $\begin{array}{l}51 \\
29 \\
27\end{array}$ \\
\hline Situación laboral de la madre y el padre & $\begin{array}{l}\text { Madre ocupada } \\
\text { Padre ocupado } \\
\text { Madre y padre ocupados }\end{array}$ & $\begin{array}{l}37 \\
78 \\
30\end{array}$ \\
\hline
\end{tabular}

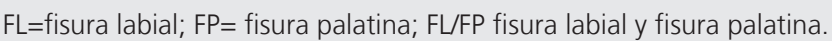




\section{Interdisciplina}

Los resultados de las variables de este indicador se muestran en la tabla 2. De los 100 niños, hubo 29 que estaban en seguimiento con las tres especialidades (FOC) y 23 que no tenían seguimiento con ninguna de las tres, predominantemente los niños con FL. Un 7\% de los niños nunca estuvo en seguimiento con fonoaudiología. De los 82 niños que se atienden en las instituciones con equipo completo, casi una cuarta parte $(n=22)$ recibió atención interdisciplinaria conjunta (los tres profesionales de FOC), al menos en el primer año del tratamiento.

\section{Oportunidad}

Para valorar la oportunidad del tratamiento, se observó la edad en que los niños comenzaron el tratamiento y la edad en que fueron intervenidos en la primera y segunda cirugía, respectivamente, y esto fue contrastado con los estándares. No hubo asociación entre haber tenido diagnóstico prenatal y comenzar tempranamente la atención, pero el inicio precoz del tratamiento sí fue más frecuente entre los niños que habían nacido en la institución tratante $(\mathrm{OR}=3,7$; IC $95 \%$ 1,4-10,1). El 30\% de los niños inició tratamiento dentro de las primeras 48 horas de vida (es decir, tuvo la primera intervención de odontología, y/o cirugía, y/o fonoaudiología), 63 niños iniciaron tratamiento en la primera semana y 5 niños luego de los tres meses de

\begin{tabular}{ll} 
Tabla 2. Características del tratamiento: Integralidad e \\
Interdisciplina, Red FLAP Argentina, 2015-2016 \\
\hline Interdisciplina & \\
Estaba en seguimiento con las especialidades básicas (FOC) & $\mathrm{n}$ \\
$\quad$ Con Fonoaudiología & 52 \\
$\quad$ Con Odontología & 55 \\
$\quad$ Con Cirugía & 54 \\
$\quad$ Con las tres & 29 \\
Modalidad de atención de las especialidades básicas FOC* & \\
$\quad$ Conjunta & 22 \\
$\quad$ En el mismo día & 48 \\
$\quad$ En días diferentes & 12 \\
Integralidad & \\
Realizó interconsulta con especialidades complementarias & \\
$\quad$ Con ORL & 74 \\
$\quad$ Con Genética & 40 \\
$\quad$ Con Servicio Social & 37 \\
$\quad$ Con Psicología & 36 \\
Control de salud pediátrico & \\
Lo realizaba habitualmente & 89 \\
Lo realizó en los últimos 6 meses & 81 \\
\hline
\end{tabular}

FOC: fonoaudiología, odontología y cirugía. ORL: otorrinolaringologia. * Se incluyen sólo a los asistidos en instituciones con equipo completo.

edad. La distribución de los niños operados (primera y segunda cirugía, respectivamente) y su condición de oportunidad, se muestran en la figura 1.

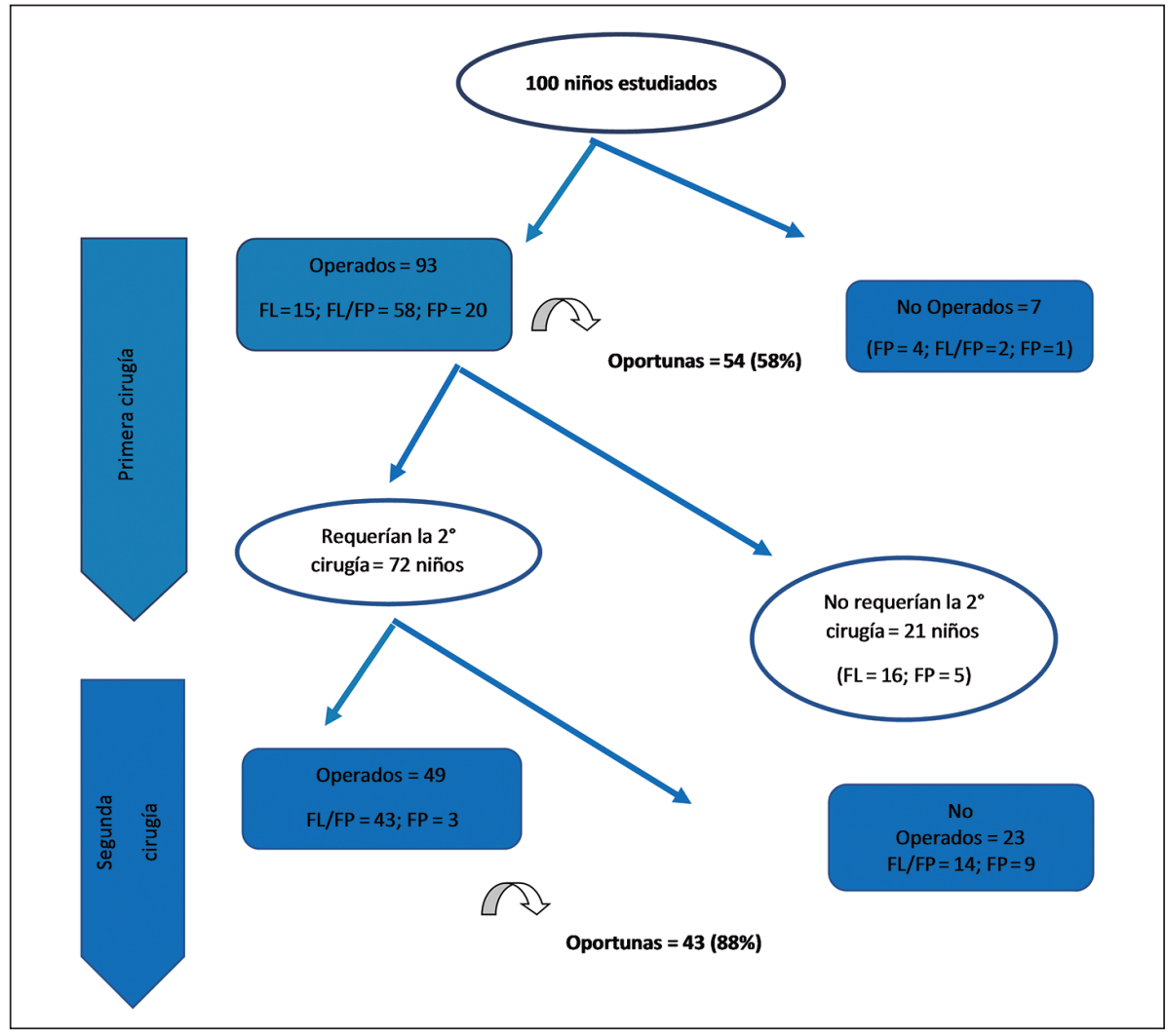

Figura 1. Oportunidad de las cirugías, niños asistidos en la Red FLAP Argentina, 2015-2016 


\section{Integralidad}

Los resultados de las variables de este indicador se muestran en la tabla 2. El 8\% de los niños nunca tuvo interconsulta con las especialidades complementarias (psicología, servicio social, genética y otorrinolaringología) y el 11\% realizó interconsulta con las cuatro. El $54 \%$ realizaba habitualmente el control pediátrico en el primer nivel de atención y 8 niños en la institución tratante.

En la tabla 3 se muestran los puntajes de cada indicador del tratamiento (interdisciplina, oportunidad e integralidad), distribuidos en categorías alta, media y baja y según el tipo de fisura.

\section{Barreras organizacionales}

Comenzaron el tratamiento en la misma institución en que nacieron 23 niños, mientras que 77 fueron derivados al nacer a otro hospital para iniciar tratamiento; en 47 de ellos, (61\%) el turno para la primera consulta fue gestionado por la Red Flap y en 30 (39\%) debió gestionarlo la familia. Para las consultas ulteriores, el $44 \%$ de las 100 familias no tuvo turnos programados, debió gestionarlos cada vez que atendieron al niño.

Habitualmente un tercio de las familias tardaba más de dos horas en llegar a la institución tratante, un $77 \%$ se trasladaba en transporte público y la mitad de éstos tomaba dos transportes.

En las tablas 4.a. 4.b. y 4.c. se muestra el análisis bivariado que relaciona qué factores del niño, la familia, o las barreras al acceso, se asociaron significativamente con las categorías (alta/media/baja) de los indicadores del tratamiento.

Tabla 3. Distribución del puntaje de los indicadores de tratamiento según categorías, por tipo de fisura, Red FLAP Argentina, 2015-2016

\begin{tabular}{|c|c|c|c|c|c|c|c|c|c|c|}
\hline \multirow[t]{2}{*}{ Tipo de fisura } & & \multicolumn{3}{|c|}{ Interdisciplina } & \multicolumn{3}{|c|}{ Oportunidad } & \multicolumn{3}{|c|}{ Integralidad } \\
\hline & & Alta & Media & Baja & Alta & Media & Baja & Alta & Media & Baja \\
\hline \multirow[t]{2}{*}{ Labio } & $n$ & 0 & 4 & 13 & 7 & 3 & 7 & 1 & 5 & 11 \\
\hline & $\%$ & & 23,5 & 76,5 & 41,2 & 17,7 & 41,2 & 10 & 29,4 & 64,7 \\
\hline \multirow[t]{2}{*}{ Labio y paladar } & $n$ & 9 & 19 & $33^{*}$ & 36 & 15 & 11 & 8 & 23 & $30 *$ \\
\hline & $\%$ & 14,8 & 31,2 & 54,1 & 58,1 & 24,2 & 17,7 & 13,1 & 37,7 & 49,2 \\
\hline \multirow[t]{2}{*}{ Paladar } & $n$ & 4 & 4 & 13 & 2 & 5 & 14 & 1 & 7 & $12^{*}$ \\
\hline & $\%$ & 19,1 & 19,1 & 61,9 & 9,5 & 23,8 & 66,7 & 10 & 35 & 60 \\
\hline Total & & 13 & 27 & 59 & 45 & 23 & 32 & 10 & 35 & 53 \\
\hline
\end{tabular}

* Sin datos $=1$ niño. Valor de $p=0,0003$.

\begin{tabular}{|c|c|c|c|c|c|}
\hline \multirow[t]{3}{*}{ Indicador } & \multirow[t]{3}{*}{ Variables asociadas } & & \multicolumn{2}{|c|}{ Puntaje } & \multirow[t]{3}{*}{ OR (IC 95\%) } \\
\hline & & & \multirow{2}{*}{$\begin{array}{c}\text { Alto/medio } \\
(\mathrm{n}=40)\end{array}$} & \multirow{2}{*}{$\begin{array}{c}\begin{array}{c}\text { Bajo* } \\
(n=59)\end{array} \\
n(\%)\end{array}$} & \\
\hline & & & & & \\
\hline \multirow[t]{8}{*}{ Interdisciplina } & Tuvo interconsulta con otorrinolaringología & Sí & $34(85,0)$ & $39(66,1)$ & $2,9(1,1-8,1)$ \\
\hline & & No & $6(15,0)$ & $20(33,9)$ & \\
\hline & Tuvo interconsulta con Servicio Social & Sí & $23(57,5)$ & $14(24,6)$ & $4,2(1,7-9,9)$ \\
\hline & & No & $17(42,5)$ & $43(75,4)$ & \\
\hline & Madre con secundario completo o más & Sí & $25(64,1)$ & $22(37,9)$ & $2,9(1,3-6,8)$ \\
\hline & & No & $14(35,9)$ & $36(62,1)$ & \\
\hline & La institución gestiona los turnos & Sí & $24(61,5)$ & $20(33,9)$ & $3,1(1,3-7,2)$ \\
\hline & & No & $15(38,5)$ & $39(66,1)$ & \\
\hline
\end{tabular}

$* \sin$ datos $=1$ niño. 
Tabla 4b. Factores asociados con un puntaje alto del indicador de Oportunidad en el tratamiento de los niños asistidos en la Red Flap Argentina, 2015-2016

\begin{tabular}{|c|c|c|c|c|c|}
\hline \multirow[t]{3}{*}{ Indicador } & \multirow[t]{3}{*}{ Variables asociadas } & & \multicolumn{2}{|c|}{ Puntaje } & \multirow[t]{3}{*}{ OR (IC 95\%) } \\
\hline & & & \multirow{2}{*}{$\begin{array}{c}\begin{array}{c}\text { Alto/medio } \\
(\mathrm{n}=45)\end{array} \\
\mathrm{n}(\%)\end{array}$} & \multirow{2}{*}{$\begin{array}{c}\text { Bajo* } \\
(n=55)\end{array}$} & \\
\hline & & & & & \\
\hline \multirow[t]{15}{*}{ Oportunidad } & En seguimiento con Fonoaudiología & Sí & $32(71,1)$ & $20(36,4)$ & $4,3(1,9-10,0)$ \\
\hline & & No & $13(28,9)$ & $35(63,6)$ & \\
\hline & En seguimiento con Odontología & Sí & $32(71,1)$ & $23(41,8)$ & $3,4(1,8-7,9)$ \\
\hline & & No & $13(28,9)$ & $32(58,2)$ & \\
\hline & Tuvo interconsulta con Psicología & Sí & $22(48,9)$ & $14(25,5)$ & $2,7(1,2-6,5)$ \\
\hline & & No & $23(51,1)$ & $41(74,6)$ & \\
\hline & Ambos padres con educación secundaria completa o más & Sí & $19(43,2)$ & $8(15,7)$ & $4,2(1,6-10,7)$ \\
\hline & & No & $25(56,8)$ & $43(84,3)$ & \\
\hline & Madre con educación secundaria completa o más & Sí & $28(62,2)$ & $19(35,9)$ & $2,9(1,3-6,7)$ \\
\hline & & No & $17(37,8)$ & $34(64,2)$ & \\
\hline & Tiempo que tarda en llegar a la institución & $\leq$ de $2 \mathrm{~h}$ & $38(84,4)$ & $32(59,3)$ & $2,9(1,4-9,9)$ \\
\hline & & $>$ de $2 \mathrm{~h}$ & $7(15,6)$ & $22(40,7)$ & \\
\hline & Tipo de Fisura* & FL/FP & $36(80,0)$ & $26(47,3)$ & $p=0,0003$ \\
\hline & & $\mathrm{FL}$ & $7(15,6)$ & $10(18,2)$ & \\
\hline & & FP & $2(4,4)$ & $19(34,6)$ & \\
\hline
\end{tabular}

FL = fisura labial; FP = fisura palatina; FL/FP fisura labial y fisura palatina.

Tabla 4c. Factores asociados con un puntaje alto/medio del indicador de Integralidad en el tratamiento de los niños asistidos en la Red FLAP Argentina, 2015-2016

\begin{tabular}{|c|c|c|c|c|c|}
\hline \multirow[t]{3}{*}{ Indicador } & \multirow[t]{3}{*}{ Variables asociadas } & & \multicolumn{2}{|c|}{ Puntaje } & \multirow[t]{3}{*}{ OR (IC 95\%) } \\
\hline & & & \multirow{2}{*}{$\begin{array}{c}\text { Alto/medio } \\
(\mathrm{n}=45)\end{array}$} & \multirow{2}{*}{$\begin{array}{c}\text { Bajo* } \\
(n=53)\end{array}$} & \\
\hline & & & & & \\
\hline \multirow[t]{18}{*}{ Integralidad } & En seguimiento con Fonoaudiología & Sí & $29(64,4)$ & $21(39,6)$ & $2,8(1,2-6,3)$ \\
\hline & & No & $16(35,6)$ & $32(60,4)$ & \\
\hline & En seguimiento con Odontología & Sí & $32(71,1)$ & $21(39,6)$ & $3,8(1,6-8,8)$ \\
\hline & & No & $13(28,9)$ & $32(60,4)$ & \\
\hline & En seguimiento con Cirugía & Sí & $31(68,9)$ & $23(43,4)$ & $2,9(1,3-6,6)$ \\
\hline & & No & $14(31,1)$ & $30(66,6)$ & \\
\hline & Las tres especialidades (FOC) asisten el mismo día & Sí & $31(68,9)$ & $17(32,7)$ & $4,6(1,9-10,7)$ \\
\hline & & No & $14(31,1)$ & $35(67,3)$ & \\
\hline & Ambos padres ocupados & Sí & $23(54,8)$ & $7(15,2)$ & $7,4(2,6-19,6)$ \\
\hline & & No & $18(45,2)$ & $39(84,8)$ & \\
\hline & Ambos padres con educación secundaria completa o más & Sí & $18(40,9)$ & $9(18,0)$ & $3,2(1,2-8,1)$ \\
\hline & & No & $26(59,1)$ & $41(82,0)$ & \\
\hline & Madre con educación secundaria completa o más & Sí & $29(64,4)$ & $17(32,7)$ & $3,7(1,6-8,7)$ \\
\hline & & No & $16(35,6)$ & $35(67,3)$ & \\
\hline & La institución gestiona los turnos & Sí & $29(64,4)$ & $18(35,3)$ & $3,3(1,4-7,7)$ \\
\hline & & No & $16(35,6)$ & $33(64,7)$ & \\
\hline & Oportunidad (Alta) & Sí & $27(60,0)$ & $17(32,1)$ & $3,2(1,4-7,3)$ \\
\hline & & No & $18(40,0)$ & $36(67,9)$ & \\
\hline
\end{tabular}

*Sin datos $=2$ niños. FOC $=$ Fonoaudiología, Odontología, Cirugía . 


\section{Discusión}

Hasta el momento, un solo estudio describió el proceso de la implementación de la Red Flap ${ }^{20}$. El presente trabajo es la primera evaluación nacional de sus resultados.

Se decidió incluir niños nacidos durante el período 2015-2016 porque todos ellos tendrían edad suficiente para ser evaluados en cuanto a las intervenciones necesarias para el cumplimiento de los tratamientos; y excluir a los niños con fisuras asociadas o sindrómicas porque sus necesidades de tratamiento y su evolución no son comparables con las correspondientes a los niños con fisuras aisladas.

La distribución proporcional del tipo de fisuras de los niños de la muestra no difiere de la reportada por la RENAC en Argentina en los últimos años ${ }^{24,25}$.

Este trabajo muestra que aunque los niños mayoritariamente se asistían en una institución con equipo básico completo, al momento de la encuesta un tercio había abandonado el seguimiento. Esto difiere de los estándares ${ }^{15,18,26}$ y contrasta con datos de países de altos ingresos como Canadá ${ }^{27}$, pero coincide con otros como Brasi $^{28}$ o México ${ }^{29}$. Los niños con FL/FP fueron los que en mayor proporción permanecían en seguimiento con FOC, mientras que los niños con FL fueron los que mayoritariamente habían perdido el seguimiento. Un estudio local que evaluó la adherencia al tratamiento de niños con FLAP, no encontró diferencias por tipo de fisura ${ }^{30}$.

Resultó satisfactorio que al menos en el primer año de tratamiento, casi el $60 \%$ de los niños que se atendían en instituciones con equipo completo, eran habitualmente asistidos por las especialidades básicas en el mismo día de consulta, aunque sólo una cuarta parte recibió atención conjunta con los tres profesionales. La atención conjunta de los equipos interdisciplinarios está fuertemente recomendada por los expertos $^{31}$. En Irán, un estudio comparó la estrategia de atención con equipo interdisciplinario de niños con FLAP aisladas, con otro grupo de similares características pero tratado por especialistas de manera individual, y encontró diferencias muy favorables en los indicadores de calidad de vida postratamiento de los primeros $^{32}$.

Las barreras de acceso estudiadas en el presente trabajo podrían explicar en parte el abandono de los tratamientos, ya que muchas familias tardan tiempos considerables para llegar al lugar de asistencia debiendo usar frecuentemente más de un trasporte. Los niños cuyas instituciones facilitan turnos programados para las consultas con FOC tuvieron mejores puntajes en interdisciplina e integralidad en el tratamiento. Al respecto, uno de los pocos estudios publicados sobre distancias y tiempos hasta los centros de atención de
FLAP en Estados Unidos, encontró que los padres no consideraban las largas distancias como "un problema", aunque ese trabajo no valoró las características o los resultados de los tratamientos recibidos ${ }^{33}$. Otro estudio analizó las barreras al tratamiento percibidas por los padres encontrando que quienes mencionaron que era muy costoso el traslado hasta el centro tratante tenían más chance de haber realizado la cirugía tardíamente $e^{34}$.

Vale la pena poner en discusión los modelos organizativos de las instituciones del subsector público. La experiencia del Reino Unido demostró que la centralización de los servicios, aumentando el volumen de niños fisurados asistidos en cada equipo, mejora los indicadores de resultado a mediano y largo plazo, objetivado a través de indicadores como el lenguaje, la apariencia física y la dentición ${ }^{35}$. En contraste, una publicación de 2018 plantea la inconsistencia de la política de regionalización y descentralización de centros tratantes implementada años atrás en Brasil, por la falta, según el autor, de instituciones capacitadas para realizar procedimientos quirúrgicos de alta compleji$\mathrm{dad}^{36}$. Lamentablemente en dicho estudio no se analiza la situación de la atención sobre datos objetivos de Brasil (cantidad de pacientes y centros asistenciales, acceso a la atención, etc.).

En el presente estudio, hubo 7 niños que no estaban operados al momento de la encuesta y 23 niños de los 72 que requerían una segunda cirugía, que no la habían concretado; estos resultados no se ajustan a las recomendaciones basadas en evidencia ${ }^{15,17,18,26}$. Es tan importante la oportunidad de la primera cirugía que se ha propuesto que la edad a la que los niños llegan a esa intervención sea utilizada como un indicador de acceso, para comparar desigualdades en los niños con FLAP entre los países y hacia el interior de ellos ${ }^{37}$.

En relación con otras publicaciones sobre la oportunidad de la cirugía, el presente trabajo encontró un número relevante de niños con puntaje alto en oportunidad. Cabe mencionar que aquí la variable inicio del tratamiento, que es el primer contacto del niño con alguna de las tres especialidades básicas (FOC), se incluyó en el indicador porque la derivación precoz del niño al equipo de tratamiento es uno de los aspectos prioritarios de la Red Flap, y por otro lado, porque -según lo establecen los estándares locales e internacionales- ${ }^{15,17,18,26}$ se conceptualizó el tratamiento, como la suma de todas las intervenciones requeridas para la rehabilitación integral del niño fisurado y no sólo la reparación quirúrgica de la malformación.

En el mismo sentido, se consideró la Interdisciplina como la modalidad de atención que, - a diferencia de la multidisciplina- implica la concurrencia de varias 
especialidades con espíritu colaborativo y una mirada compartida, centrada en el paciente ${ }^{31}$. Asimismo, en consonancia con los centros tratantes con vasta experiencia internacional en FLAP, para definir integralidad se focalizó en la responsabilidad del pediatra en el seguimiento longitudinal de la salud del niño, mediante el control de salud y la interconsulta con las especialidades complementarias, para detectar y tratar a tiempo las comorbilidades que acompañan a las fisura orales ${ }^{38,39}$.

El control pediátrico regular y actualizado fue referido por el $90 \%$ de los padres, el dato es similar al de la población general de niños en Argentina ${ }^{40}$. A pesar de que fue alta la proporción de niños en seguimiento pediátrico y que el pediatra tiene un rol central en motivar a los padres para sostener el seguimiento, no se acompañó de la misma proporción de casos con cumplimiento de los indicadores.

Finalmente, los únicos factores que se asociaron significativamente con los puntajes altos de los tres indicadores del tratamiento fueron las características sociales de las familias. Tener padres ocupados o con mayor nivel educativo, permitió a los niños fisurados acceder a un tratamiento con mejores indicadores. Se ha postulado que las familias con características sociales desfavorables tienen mayor riesgo de concebir un hijo fisurado ${ }^{7,8}$, por lo tanto, si esas familias tienen un niño con FLAP, enfrentan una doble inequidad.

La principal debilidad del estudio es la común a las encuestas telefónicas: pudo haber un sesgo de selección de sujetos que resultaron más fáciles de contactar, al excluir a los que no tienen teléfono, o viven en zonas con escasa señal telefónica. No obstante, algunos de los niños incluidos en el trabajo, fueron contactados por intermedio de vecinos o familiares, por lo que, pudieron estudiarse familias que no tenían teléfono propio. Por otro lado, por la edad de los niños, podría existir un sesgo de recuerdo de parte de sus madres en los datos de los primeros meses de vida. No obstante, las madres suelen recordar muy bien los eventos de la salud de sus hijos, lo que se ha estudiado comparándolo con registros médicos ${ }^{41}$. El reporte materno de encuestas en hogares es una buena fuente de información independientemente de características maternas como la edad, el número de hijos, o las condiciones de vida ${ }^{42}$.

Como fortaleza, el instrumento permitió una muestra representativa de la Red Flap de todas las regiones de Argentina, con un costo reducido en tiempo y dinero, y logró "encontrar" familias que quizás no se hubiera podido convocar desde los servicios.

Las escasas publicaciones nacionales sobre resultados del tratamiento de niños con fisuras orales describen mayormente resultados estéticos o las innovaciones en materia de técnicas quirúrgicas ${ }^{43}$. En el presente trabajo se presentan datos de todo el proceso del tratamiento, desde la captación del RN, su derivación a los centros tratantes y las características del seguimiento en los primeros años de la niñez. Sería importante profundizar en investigaciones que describan los resultados a largo plazo de estos niños, evaluando su inserción social, al ingreso escolar.

En Argentina aunque la atención en los efectores del subsector público es gratuita para toda la población, la oferta de servicios no es uniforme. La extensión territorial dificulta a la población con menos recursos el acceso a la atención especializada. Para los RN con anomalías congénitas la situación es aún más dramática, ya que frecuentemente necesitan profesionales altamente especializados, con vasta experiencia para tratarlos. La política de regionalización de la atención perinatal ha logrado mejoras en la atención de los neonatos de riesgo, especialmente los prematuros y/o con bajo peso ${ }^{44}$. La atención en red de los $\mathrm{RN}$ con anomalías congénitas requiere un mayor desarrollo en el futuro inmediato.

En conclusión, aunque los niños con FLAP realizaron controles de salud periódicos y se asistieron en instituciones con equipos multidisciplinarios, la mitad recibió tratamiento con puntaje alto y medio para los indicadores de interdisciplina e integralidad, y dos tercios tuvieron tratamiento con alto puntaje de oportunidad. Aún existen barreras que dificultan el acceso a la atención, como las grandes distancias o la gestión de los turnos. En las familias con una menor vulnerabilidad social, fundamentalmente conformadas por padres que tienen trabajo y un nivel educativo superior, la proporción de niños que recibieron un tratamiento acorde a los estándares fue mayor.

\section{Responsabilidades Éticas}

Protección de personas y animales: Los autores declaran que los procedimientos seguidos se conformaron a las normas éticas del comité de experimentación humana responsable y de acuerdo con la Asociación Médica Mundial y la Declaración de Helsinki.

Confidencialidad de los datos: Los autores declaran que han seguido los protocolos de su centro de trabajo sobre la publicación de datos de pacientes.

Derecho a la privacidad y consentimiento informado: Los autores han obtenido el consentimiento informado de los pacientes y/o sujetos referidos en el artículo. Este documento obra en poder del autor de correspondencia. 


\section{Financiamiento}

El trabajo ha sido financiado parcialmente por la Dirección de Investigación en Salud, Ministerio de Salud (Argentina), a través una Beca "Salud Investiga-Abraam Sonis", convocatoria 2018 y el Instituto de Inves- tigaciones Epidemiológicas, Academia Nacional de Medicina.

\section{Conflicto de intereses}

Los autores declaran no tener conflicto de intereses.

\begin{tabular}{|c|c|c|c|c|}
\hline Indicador & Variables & Categorías & Valor & Puntaje \\
\hline \multirow{6}{*}{ Interdisciplina } & \multirow{3}{*}{$\begin{array}{l}\text { Modalidad de atenciónde las tres } \\
\text { especialidades básicas (FOC) }\end{array}$} & $\begin{array}{l}\text { Las consultas se realizaron en la misma institución, en } \\
\text { el mismo momento (consulta conjunta) }\end{array}$ & 2 & \multirow{6}{*}{$\begin{array}{c}\text { Alta: } \\
\text { 7-6 } \\
\text { Media: } \\
\text { 5-4 } \\
\text { Baja: } \\
<4\end{array}$} \\
\hline & & $\begin{array}{l}\text { Las consultas se realizaron en la misma institución, el } \\
\text { mismo día de atención }\end{array}$ & 1 & \\
\hline & & $\begin{array}{l}\text { Las consultas se realizaron en la misma institución } \\
\text { pero en días diferentes. }\end{array}$ & 1 & \\
\hline & \multirow{3}{*}{$\begin{array}{l}\text { Se encuentra actualmente en } \\
\text { seguimiento }\end{array}$} & Con las tres especialidades básicas (FOC) & 3 & \\
\hline & & Con dos especialidades básicas & 2 & \\
\hline & & Con una especialidad básica & 1 & \\
\hline \multirow{10}{*}{ Oportunidad } & \multirow{4}{*}{$\begin{array}{l}\text { Comienzo del tratamiento con } \\
\text { equipo (FOC) }\end{array}$} & En las primeras 48 horas & 3 & \multirow{4}{*}{$\begin{array}{l}\text { Alta: } \\
\text { 9-7 }\end{array}$} \\
\hline & & Entre los 3 y 7 días de vida & 2 & \\
\hline & & Entre los 8 y 15 días de vida & 1 & \\
\hline & & Luego de los 15 días & 0 & \\
\hline & \multirow{4}{*}{$\begin{array}{l}\text { Cirugía para FL/FP } \\
\text { o FP }\end{array}$} & Se realizó la primera cirugía & 2 & \multirow{4}{*}{$\begin{array}{c}\text { Media: } \\
6-5 \\
\text { Baja: } \\
<5\end{array}$} \\
\hline & & $\begin{array}{l}\text { Primera cirugía oportuna: (edad del niño acorde a las } \\
\text { normativas) }\end{array}$ & 1 & \\
\hline & & Se realizó la segunda cirugía & 2 & \\
\hline & & $\begin{array}{l}\text { Segunda cirugía oportuna: (edad del niño acorde a las } \\
\text { normativas) }\end{array}$ & 1 & \\
\hline & \multirow{2}{*}{$\begin{array}{l}\text { Cirugía para FL o niños sin indicación } \\
\text { de segunda cirugía }\end{array}$} & Se realizó la primera cirugía & 2 & \multirow{2}{*}{$\begin{array}{l}\text { Alta: } 6 \\
\text { Media: } 5 \\
\text { Baja }<5\end{array}$} \\
\hline & & $\begin{array}{l}\text { Primera cirugía oportuna: (edad del niño acorde a las } \\
\text { normativas }\end{array}$ & 1 & \\
\hline \multirow{9}{*}{ Integralidad } & \multirow{4}{*}{$\begin{array}{l}\text { Interconsulta con las especialidades } \\
\text { complementarias (Psicología, Servicio } \\
\text { Social, Genética, ORL) }\end{array}$} & 1 especialidad & 1 & \multirow{9}{*}{$\begin{array}{c}\text { Alta: } 7 \\
\text { Media: 6-5 } \\
\text { Baja }<5\end{array}$} \\
\hline & & 2 especialidades & 2 & \\
\hline & & 3 especialidades & 3 & \\
\hline & & 4 especialidades & 4 & \\
\hline & \multirow{2}{*}{$\begin{array}{l}\text { Realiza habitualmente control } \\
\text { pediátrico }\end{array}$} & $\mathrm{Si}$ & 1 & \\
\hline & & No & 0 & \\
\hline & \multirow{3}{*}{ Realizó el último control pediátrico } & Hace $<$ de 6 meses & 2 & \\
\hline & & Entre 6 a 12 meses & 1 & \\
\hline & & Más de 12 meses & 0 & \\
\hline
\end{tabular}




\section{Referencias}

1. Wyszynski D, Duffy D BT. Maternal cigarette smoking and oral clefts. a meta-analysis. Cleft Palate-Craniofacial J. 1997;34(3):206-10.

2. Kallen K. Maternal smoking and orofacial clefts. Cleft Palate-Craniofacial J. 1997;34(1):11-6.

3. Leite ICG, Koifman S. Oral clefts, consanguinity, parental tobacco and alcohol use: a case-control study in Rio de Janeiro, Brazil. Braz Oral Res [Internet]. 2009;23(1):31-7. Available from: http://www.ncbi.nlm.nih.gov/ pubmed/19488469

4. De Queiroz Correa Herkrath A, Herkrath F, Besa Rebelo M, Vianna Vettore M. Parental age as a risk factor for nonsyndromic oral clefts: A meta-analysis. J Dent. 2012;40(1):3-14.

5. Berg E, Lie RT, Sivertsen A, Haaland ØA. Parental age and the risk of isolated cleft lip: A registry-based study. Ann Epidemiol [Internet]. 2015;25(12):942-7. Available from: http://dx.doi.org/10.1016/j. annepidem.2015.05.003

6. Sabbagh HJ, Hassan MHA, Innes NPT, Al Baik A, Mossey PA. Parental consanguinity and nonsyndromic orofacial clefts in children: A systematic review and meta-analyses. Cleft PalateCraniofacial J. 2014;51(5):501-13.

7. Lupo PJ, Danysh HE, Symanski E, Langlois PH, Cai Y, Swartz MD. Neighborhood-based socioeconomic position and risk of oral clefts among offspring. Am J Public Health. 2015;105(12):2518-25.

8. Escoffié-Ramirez M, Medina-Solís CE, Pontigo-Loyola AP, Acuña-González G, Casanova-Rosado JF, Colome-Ruiz GE. Asociación de labio y/o paladar hendido con variables de posición socioeconómica: un estudio de casos y controles. Rev Bras Saúde Matern Infant [Internet]. 2010;10(3):323-9. Available from: http:// search.ebscohost.com/login.aspx?direct= true $\& \mathrm{db}=$ asx \&AN $=57192850$ \&site $=$ eds live

9. Prescott NJ, Sue M. Folate and the face: Evaluating the evidence for the influence of folate genes on craniofacial development. Cleft Palate-Craniofacial J. 2002;39(3):327-31.

10. Vieira-Machado CD, de Carvalho FM, Santana da Silva LC, et al. Analysis of the genetic ancestry of patients with oral clefts from South American admixed populations. Eur J Oral Sci. 2016;124(4):406-11.

11. Barbero P, Bidondo M, Duarte $S$, Groisman B, Liascovich R, Piola A. Reporte Anual RENAC 2019 [Internet]. 2019. [citado el 13 de junio de 2020]. Available from: http://www.anlis.gov.ar/ cenagem/wp-content/uploads/2017/07/ Reporte-RENAC-2019.pdf

12. Christensen K, Mortensen PB. Facial clefting and psychiatric diseases: A follow-up of the Danish 1936-1987 Facial Cleft Cohort. Cleft Palate-Craniofacial J. 2002;39(4):392-6.

13. Wehby GL, Collett BR, Barron S, Romitti $\mathrm{P}$, Ansley T. Children with oral clefts are at greater risk for persistent low achievement in school than classmates. Arch Dis Child. 2015;100(12):1148-54.

14. Wehby GL, Moreno LM. Oral clefts and wellbeing: A lifelong perspective and an agenda for comparative effectiveness research. J Comp Eff Res. 2014;3(1):23-8.

15. Ministerio de salud. Guía Clínica AUGE: Fisura Labiopalatina. Minist Salud Pública Chile [Internet]. 2015 [citado el 13 de junio de 2020];3:1-97. Available from: http://www.bibliotecaminsal.cl/ wp/wp-content/uploads/2016/04/guiaFisuraLabioPalatina-2015-CM.pdf

16. Instituto de Seguridad Social de Uruguay. Guia Clinica Diagnostico y Tratamiento Fisura Labio Alveolo Palatina [Internet]. 2014 [citado el 13 de junio de 2020]. Available from: https://www.bps.gub. uy/bps/file/13116/1/guia_clinica_fisura_ labio_alveolo_palatina.pdf

17. Equipo Multidisciplinario Integral Hospital Nacional Prof. Alejandro. Guía para la Atención de Pacientes con Fisuras Naso-Labio-Alveolo-Palatinas [Internet]. Haedo; 2010 [citado el 7 de julio de 2020]. p. 1-57. Available from: https://www.yumpu.com/es/document/ view/13452413/descargar-la-guia-clinicapara-el-tratamiento-de-hospital-posadas

18. CEN. Early care services for babies born wit cleft lip and/or palate. Cen [Internet]. 2015; [citado el 13 de junio de 2020]. Available from: http://europeancleft.org/ wp-content/uploads/2016/02/CEN.pdf

19. Andrade J, Chuit R, Consoli N, et al. Análisis Epidemiológico de 970 pacientes con fisura labio-alveolo-palatina con tratamiento pre-quirúrgico y quirúrgico, Argentina 2009. Bol A N Med. 2009;87(2) (ISSN 0374-647 X):261-272.

20. Cassinelli A, Pauselli N, Piola A, et al. Red nacional para la atención de niños/ as con fisuras orales: organización, funcionamiento y primeros resultados. Arch Argent Pediatr [Internet]. 2018;116(1):e26-33. Available from: http://www.sap.org.ar/docs/publicaciones/ archivosarg/2018/v116n1a14.pdf

21. Indec. Encuesta Permanente de Hogares [Internet]. INDEC. Instituto Nacional de Estadística y Censos. 2019 [citado el 13 de junio de 2020]. p. 33. Available from: tps://www.indec.gob.ar/ftp/cuadros/ menusuperior/eph/EPH_registro_t318. pdf

22. Ministerio de Salud y Desarrollo Social.
Sistema Informático Perinatal para la Gestión (SIP-G) Indicadores básicos 2017 República Argentina [Internet]. Buenos Aires; 2018. [citado el 13 de junio de 2020] Available from: tps://www.indec. gob.ar/ftp/cuadros/menusuperior/eph/ EPH_registro_t318.pdf

23. Seid M, Opipari-Arrigan L, Gelhard LR, Varni JW, Driscoll K. Barriers to Care Questionnaire: Reliability, Validity, and Responsiveness to Change Among Parents of Children With Asthma. Acad Pediatr [Internet]. 2009;9(2):106-13. Available from: http://dx.doi.org/10.1016/j. acap.2008.12.003

24. Groisman B, Tardivo A, Gili J, et al. Reporte Anual RENAC 2015 [Internet]. 2015. [citado el 13 de junio de 2020] Available from: http://www.anlis.gov.ar/ cenagem/wp-content/uploads/2017/07/ Reporte-Renac-2015-web.pdf

25. Groisman B, Duarte S, Gili J, et al. Reporte Anual RENAC 2016 [Internet]. 2016. [citado el 13 de junio de 2020] Available from: http://www.anlis.gov.ar/ cenagem/wp-content/uploads/2017/07/ Reporte-Renac-2016-web.pdf

26. Association ACP-C. Standards for Approval of Cleft Palate and Craniofacial Teams-Commission on Approval of Teams. 2019. www. acpa-cpf. org. Stand pdf [Internet]. 2019; [citado el 7 de julio de 2020] Available from: https://acpa-cpf. org/team-care/standardscat/standards-ofapproval-for-team-care/

27. Matthews JLK, Oddone-Paolucci E, Harrop RA. The epidemiology of cleft lip and palate in Canada, 1998 to 2007. Cleft Palate-Craniofacial J. 2015;52(4):417-24.

28. Silva HPV da, Arruda TTS, Souza KSC de, et al. Risk factors and comorbidities in Brazilian patients with orofacial clefts. Braz Oral Res. 2018;32(0):1-12.

29. Pons-Bonals A, Pons-Bonals L, HidalgoMartínez SM, Sosa-Ferreyra CF. Clinical-epidemiological study in children with cleft lip palate in a secondary-level hospital. Bol Med Hosp Infant Mex [Internet]. 2017;74(2):107-21. Available from: http://dx.doi.org/10.1016/j. bmhimx.2016.11.008

30. Andrade J, Chuit R, Infante L, Ling C, Miranda T, Nalda F. Adherencia al tratamiento del paciente con fisura labiopalatina (FLAP) 2006-2013. Bol AN Med. 2014;92(2):281-90.

31. Murphy MT. Collaborative interdisciplinary agreements: A new paradigm in laboratory and specialist communication and patient care. J Am Dent Assoc. 2006;137(8):1164-7.

32. Khanchezar F, Moradi N, Tahmasebi Fard N, Latifi SM, Bassak Nejad S, Hosseini Beidokhti M. The Effect of Teamwork on Children With Cleft Lip and Palate and Their Mother's Quality of Life. 
Cleft Palate-Craniofacial J [Internet]. 2019;105566561985374. Available from: http://journals.sagepub.com/ doi/10.1177/1055665619853749

33. Cassell CH, Strassle P, Mendez DD, et al. Barriers to care for children with orofacial clefts in North Carolina. Birth Defects Res Part A - Clin Mol Teratol. 2014;100(11):837-47.

34. Ise A, Menezes C, Neto JB, et al. PatientPerceived Barriers to Accessing Cleft Care at a Tertiary Referral Center in Sao Paulo, Brazil. Cleft Palate Craniofac J. 2019;56(5):639-645.

35. Ness AR, Wills AK, Waylen A, et al. Centralization of cleft care in the UK. Part 6: A tale of two studies. Orthod Craniofacial Res. 2015;18:56-62.

36. Tovani Palone M, Treatment of Nonsyndromic Cleft Lip and/or Palate in Brazil: Existing Consensus and Legislation, Scope of the Unified Health System, Inconsistencies and
Future Perspectives. World Health and Population. 2018; 17(4): 31-36.

37. Mossey PA, Shaw WC, Munger RG, Murray JC, Murthy J, Little J. Global oral health inequalities: challenges in the prevention and management of orofacial clefts and potential solutions. Adv Dent Res. 2011;23(2):247-58.

38. Lewis CW, Jacob LS, Lehmann CU. The Primary Care Pediatrician and the Care of Children With Cleft Lip and/or Cleft Palate. Pediatrics. 2017;139(5):e20170628.

39. Lombardo-Aburto E. The intervention of the pediatrician in the child with cleft lip and palate. Acta Pediatr Mex. 2017;38(4):267-73.

40. Ministerio de Salud. Indicadores seleccionados de salud y nutrición población materno-infantil [Internet]. 2019. [citado el 13 de junio de 2020] Available from: http://www. msal.gob.ar/images/stories/bes/ graficos/0000001785cnt-ennys-materno- infantil.pdf

41. Pless C, Pless B. How well they remember. Arch Pediatr Adolesc Med. 1995;149:553558.

42. D'Souza-Vazirani D, Minkovitz C, Strobino D. Validity of maternal report of acute health care use for children younger than 3 years. Arch Pediatr Adolesc Med. 2005;159(2):167-72.

43. Bennun RD, Langsam AC. Long-term results after using dynamic presurgical nasoalveolar remodeling technique in patients with unilateral and bilateral cleft lips and palates. J Craniofac Surg. 2009;

44. De Maucio B, Fecina R, Schwarcz A, Garibaldi M, Mendez Valdemarin G. Regionalización de la Atención Perinatal [Internet]. Ministerio de Salud. 2011 [citado el 13 de junio de 2020]. p. 119. Available from: http:// www.msal.gob.ar/images/stories/ bes/graficos/0000000176cnt-n02regionalizacion.pdf 